\title{
DETECÇÃO DE ÁRVORES EM NUVENS DE PONTOS DE VARREDURA LASER TERRESTRE
}

\section{Tree detection in point clouds derived from terrestrial laser scanning}

\author{
André Leonardo Bortolotto Buck 1 \\ Christel Lingnau ${ }^{2}$ \\ Álvaro Muriel Lima Machado ${ }^{3}$ \\ Sylvio Péllico Netto ${ }^{2}$
}

1 Universidade Federal do Paraná, Programa de Pós-Graduação em Engenharia Florestal (UFPR) Curitiba, PR, Brasil - andrebuck.sf@gmail.com

2 Universidade Federal do Paraná, Departamento de Ciências Florestais (UFPR) - Curitiba, PR, Brasil lingnau@ufpr.br, sylviopelliconetto@gmail.com

3 Universidade Federal do Paraná, Departamento de Geomática (UFPR) - Curitiba, PR, Brasil alvaroml@ufpr.br

\section{Resumo:}

A utilização do laser terrestre para levantamentos em povoamentos florestais tem como objetivo prover dados à modelagem tridimensional das árvores, no entanto, para que seja possível aplicar tal modelo, é necessário realizar a detecção dos pontos que fazem parte de árvores na varredura. O presente estudo propõe um método para a detecção de árvores a partir da nuvem de pontos 3D de plantios florestais. Inicialmente, procura-se reconstituir a distribuição espacial das árvores a partir da aplicação de um algoritmo de segmentação em uma seção transversal (1 metro) da nuvem de pontos. Em seguida, é apresentado um algoritmo para detectar a posição das árvores com base no padrão de alinhamento do povoamento. Por fim, os resultados obtidos são apresentados para validação pelo usuário da nuvem de pontos. O método apresentado foi testado em parcelas circulares instaladas em povoamentos de Eucalyptus spp. levantados por varreduras simples e múltiplas. Os resultados apontaram a necessidade de utilização de múltiplas estações de TLS para redução do efeito de sombreamento no levantamento das parcelas circulares. A aplicação do método de detecção de árvores em conjunto com a análise visual resultou na identificação de $100 \%$ das árvores a partir das nuvens de pontos das parcelas.

Palavras-chave: plantios florestais, varredura simples e múltiplas, parcelas circulares, componentes conectadas, Eucalyptus spp.

\begin{abstract}
:
Terrestrial LIDAR measurements in forest stands is often used to gather data for 3D tree models. However, such models require the detection of points representing trees in the scanning field. The present study offers a method for tree detection from a 3D point cloud of forest plantations. Initially the spatial distribution of trees is reconstructed by applying a segmentation algorithm in a transverse slice ( 1 meter) through the point cloud. This is followed by an algorithm for detecting tree position based on plantation stand row alignment. Finally, the results are presented
\end{abstract}


for validation by the point cloud user. The methods were evaluated over young Eucalyptus spp. stands (i.e. 2, 4 and 5 years) exploring single and multiple positioning of the TLS device inside the circular plots. Results suggest that several TLS stations should be used to reduce shading effects in mapping circular plots. Employing the tree detection method with the visual analysis of point clouds of each plot were identified $100 \%$ of the trees.

Keywords: forest plantations, single and multiple scan, circular plots, connect components, Eucalyptus spp.

\section{Introdução}

As técnicas biométricas para quantificar povoamentos florestais são bem conhecidas e vêm sendo utilizadas com frequência pelas empresas do setor. No entanto, a obtenção de medidas acuradas e estimativas precisas tem sido o grande desafio imposto ao planejamento da produção florestal.

A aplicação da varredura laser terrestre (TLS) é uma alternativa para obtenção de variáveis dendrométricas em relação aos métodos clássicos de mensuração florestal. A condição essencial para ampla aplicação dessa tecnologia é a implementação de algoritmos que permitam a automatização das medições a serem realizadas nas parcelas (Zasada et al., 2013).

Os levantamentos por TLS podem ser caracterizados pela riqueza de detalhes dos objetos encontrados no campo de visada da estação no momento da varredura. A desvantagem é a de que os pontos não são identificados quanto ao tipo de objeto refletido, apenas as coordenadas (XYZ) e o valor de intensidade são registrados (Simonse et al., 2003).

Dessa forma, para aquisição de informações a partir de nuvens de pontos de levantamentos em povoamentos florestais, detectar árvores e seu respectivo posicionamento constitui uma etapa essencial para obtenção de variáveis dendrométricas, tais como: diâmetros, alturas e volumes individuais de troncos (Aschoff e Spiecker, 2004).

A detecção de árvores em nuvem de pontos é um procedimento adotado com objetivo de determinar a localização espacial de árvores que serão submetidas à modelagem individual para a obtenção de variáveis dendrométricas. Os algoritmos para automatização desse procedimento devem ser flexíveis e proporcionar resultados condizentes com a realidade de campo.

Simonse et al. (2003), Aschoff e Spiecker, (2004), Aschoff et al. (2004) aplicaram a transformada de Hough em imagens geradas a partir da projeção de recortes da nuvem de pontos em determinada altura do solo com uma espessura de $10 \mathrm{~cm}$ para identificar as seções transversais do tronco de árvores. No entanto, o método não é robusto e pode resultar em erros na detecção (Schilling et al., 2011)

Uma abordagem que não requer a transformação dos dados em imagens para detecção de troncos foi proposta por Liang et al. (2012) para varreduras simples e Liang e Hyypä (2013) para varreduras múltiplas. Inicialmente, a distribuição espacial dos pontos é trabalhada pela análise de componentes principais para detectar agrupamentos de pontos que representam o tronco da árvore. Em seguida, o tronco é modelado por meio do ajuste sucessivo de cilindros. Os resultados apontaram um incremento na taxa de detecção de árvores em varreduras múltiplas. 
A utilização de uma seção, compreendida entre 1 e 2 metros acima da superfície do terreno, para definir agrupamento de pontos foi proposta por Brolly e Kiraly, (2009). O método utiliza o algoritmo k-means modificado para gerar agrupamentos com base nos seguintes critérios: mínimo de pontos e raio máximo por agrupamento. Os agrupamentos que satisfazem as condições são classificados como candidatos a tronco.

A disposição de dados em estruturas tridimensionais não hierárquicas ou hierárquicas tem simplificado o processamento de nuvens de pontos. Estruturas de dados não-hierárquicas são organizadas em um matriz 3D discreta particionada em cubos de igual tamanho, que podem ser chamadas de voxel space. Já as estruturas hierárquicas baseiam-se na subdivisão recursiva de uma região ou espaço, onde a partição tridimensional é executada com um Octree (Bienert et al., 2010).

A análise de nuvem de pontos em voxel space foi proposta por Gorte and Pfeifer (2004). Posteriormente, Gorte e Winterhalder (2004) apresentaram uma adaptação do algoritmo denominado Connected component labelling, comumente aplicado na análise digital de imagens (raster), para segmentar troncos e galhos de uma árvore. A partir da análise de vizinhos em espaço tridimensional não hierárquica os autores alcançaram a separação de árvores em povoamentos de coníferas com dossel aberto (Bienert et al., 2010).

Em uma estrutura hierárquica gerada por Octree a análise da topologia dos objetos representados em nuvens de pontos foi realizada por Bucksch e Wageningen (2006). A partir da análise de grafos formados pela conectividade das células e de suas principais direções cartesianas, Bucksch et al. (2010) propuseram o método SkelTre para reconstrução da árvore em tronco e galhos.

Os plantios florestais comerciais, em geral, são formados por árvores que foram intencionalmente dispostas em um padrão regular de distribuição espacial. Tal condição pode ser explorada no desenvolvimento de um algoritmo para a detecção de árvores baseado no padrão de alinhamento do povoamento. O presente estudo propõe um método para detectar árvores a partir da nuvem de pontos de TLS em plantios florestais. Inicialmente, o recorte de uma seção transversal da nuvem de pontos é submetido à segmentação por componentes conectadas em uma estrutura Octree. A partir de cada subconjunto gerado são extraídos os vetores de médias e aplicado um algoritmo para análise do alinhamento de árvores. Por fim, os resultados obtidos são apresentados para validação pelo usuário da nuvem de pontos.

\section{Materiais e métodos}

\section{1 Área de estudo}

A área de estudo está localizada no município de Três Lagoas, Estado do Mato Grosso do Sul, em povoamentos comerciais implantados no espaçamento de 3,60 x 2,20 metros, o que equivale a uma densidade de 1262 árvores/ha, conduzidos para o corte raso ao final de um ciclo de 7 anos. O levantamento foi realizado em parcelas circulares de $400 \mathrm{~m}^{2}$, contemplando povoamentos clonais de Eucalyptus spp. em três idades: 2; 4 e 5 anos. Em campo as árvores das parcelas 
foram contadas, numeradas e demarcadas com tinta e fita branca. Em cada idade foram instaladas quatro parcelas. $\mathrm{O}$ sub-bosque pode ser caracterizado pela presença de vegetação rasteira (gramíneas) e por pequenos arbustos, encontrados com maior frequência nos povoamentos de 4 e 5 anos de idade. O relevo é plano à suave ondulado, sendo que a declividade média na área das parcelas foi de aproximadamente $6 \%$.

\subsection{Varredura laser terrestre}

O equipamento Trimble TX5 foi utilizado para a reconstituição da área das parcelas circulares instaladas em campo (Figura 1 - A). A resolução das varreduras foi fixada em 1/4, correspondendo a uma resolução angular de $0,036^{\circ}$ e uma resolução espacial de $6 \mathrm{~mm}$ para uma distância de $10 \mathrm{~m}$ do equipamento. A taxa de aquisição de dados foi de 122.000 pontos/segundo. Cinco alvos esféricos de $145 \mathrm{~mm}$ de diâmetro e coloração branca foram posicionados em tripés dentro da área da parcela para auxiliar na etapa de registro das varreduras múltiplas. A instalação dos alvos não seguiu um alinhamento específico, o requisito foi que pelo menos três alvos fossem contemplados a partir de cada estação (Figura 1- B).

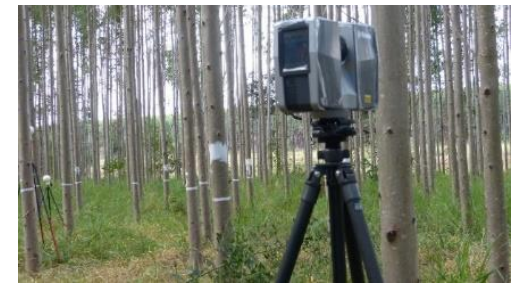

(A)

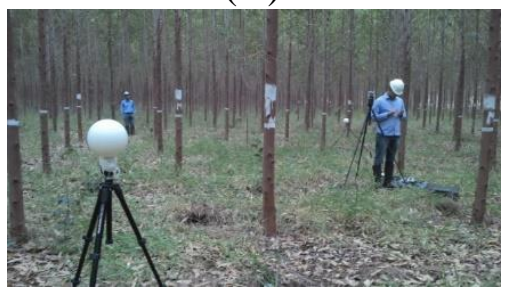

(B)

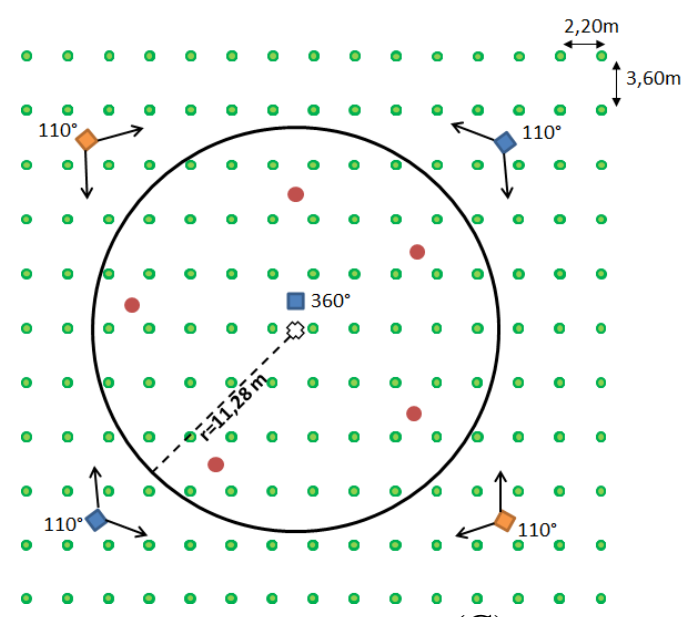

(C)
\3 CENTRO DA PARCELA

- - RAIO DA PARCELA (r)

O parcela

ÁRVORES

- ALVOS ESFÉRICOS

$\square$ TLS

$\square$ TLS AUXILIAR

Figura 1: Levantamento por varredura laser terrestre: A) Laser terrestre; B) Alvos esféricos instalados na área da parcela; C) Disposição das estações na parcela circular

As estações foram dispostas da seguinte forma (Figura 1 - C): uma estação de referência instalada no centro da parcela, na qual o campo de visão foi de $360^{\circ}$ (horizontal) x $300^{\circ}$ (vertical) com registros fotográficos, e demais estações alocadas externamente ao perímetro da parcela com campo de visada de $110^{\circ}$ (horizontal) x $300^{\circ}$ (vertical). 


\subsection{Pré-processamento de dados}

As parcelas levantadas em campo por múltiplas estações de TLS foram reconstituídas em duas formas distintas: nuvem de pontos de varredura simples e nuvem de pontos de varredura múltipla. A nuvem de pontos de varredura simples foi gerada apenas com os dados da estação de referência (varredura central). A nuvem de pontos de varredura múltipla foi gerada a partir da união das múltiplas varreduras adotando-se como referência os alvos instalados nas parcelas. Para isso as múltiplas varreduras foram submetidas ao procedimento de registro utilizando a transformação de Helmert (também conhecida como isogonal ou conforme). Os dados foram trabalhados em um sistema de coordenadas local, definido a partir da orientação da estação utilizada no levantamento da varredura de referência. Para facilitar a análise visual das nuvens de pontos de varredura simples e múltiplas, cada ponto das varreduras de referência (varredura central) teve atribuído uma cor baseada no valor RGB do pixel das fotografias obtidas pelo equipamento. A delimitação das parcelas também foi realizada para que os dados contidos na nuvem de pontos de cada levantamento representassem apenas a área das parcelas em campo.

\subsection{Detecção de árvores em nuvem de pontos de povoamentos florestais}

A automatização da detecção de árvores apresentada neste estudo está fundamentada na determinação da direção das linhas de plantio em nuvens de pontos de povoamentos florestais levantados por TLS. Para tal, o processamento da nuvem de pontos da parcela está estruturado em três etapas: 1) Recorte de uma seção transversal da nuvem de pontos; 2) Segmentação por componentes conectadas; 3 ) Aplicação do algoritmo Tree Detecion in Plantation.

1) Recorte de uma seção transversal da nuvem de pontos: A nuvem de pontos de cada parcela foi submetida a um recorte em uma seção de dados referenciados no eixo "Z" (Simonse et al., 2003; Aschoff et al., 2004; Bienert et al., 2006; Bienert et al., 2007) para representação de parte do tronco das árvores em um plantio (Figura 2).

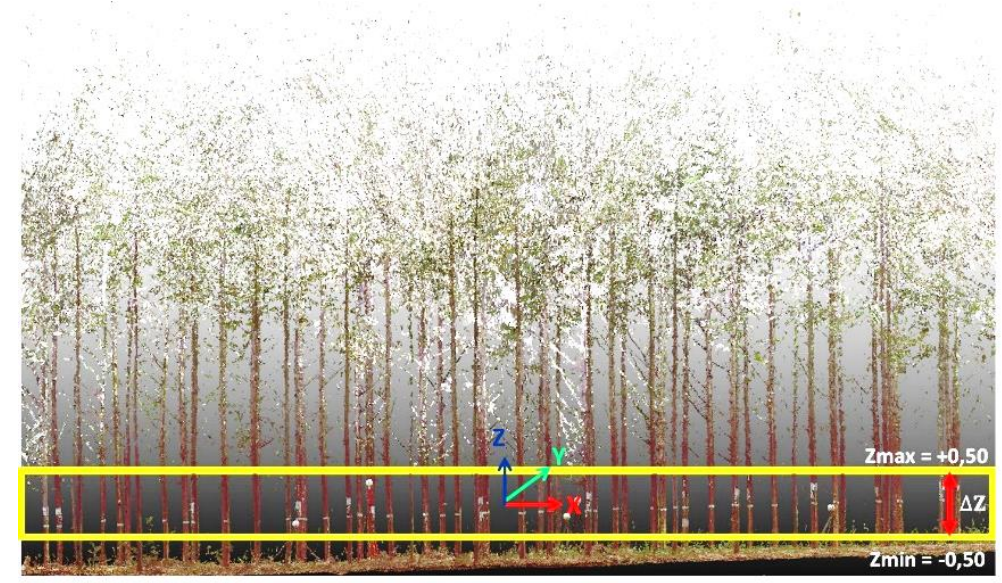

Figura 2: Recorte da seção transversal da nuvem de pontos de uma parcela 
$\mathrm{O}$ intervalo estabelecido para esta seção foi padronizado em um metro, sendo $0,50 \mathrm{~m}$ acima $\left(Z_{\text {max }}\right)$ e abaixo $\left(Z_{\text {min }}\right)$ da origem $(Z=0)$ do sistema de coordenadas local. A escolha do intervalo de um metro permitiu que os troncos fossem distinguidos na nuvem de pontos pela forma geométrica de um cilindro (Brolly e Király, 2009). A demarcação das árvores, realizada durante a coleta de dados, possibilitou a validação dos resultados.

2) Segmentação por componentes conectadas: A segmentação da seção da nuvem de pontos foi alcançada por meio do algoritmo de segmentação por componentes conectadas, proposto por Girardeau-Montaut (2006) e denominado "Label Connected Components".

O algoritmo utiliza a estrutura Octree (Meagher, 1982) para extrair conexões entre os pontos utilizando dois parâmetros de entrada: 1) Octree level; 2) Min. Points. O primeiro define o número de subdivisões do Octree, por padrão foram utilizadas oito subdivisões. O segundo restringe o número de pontos a uma quantidade mínima por componente, e quando a condição não é satisfeita, a componente é ignorada. No presente estudo esse parâmetro foi definido com base na resolução utilizada para o levantamento por TLS e na superfície de um tronco de cilindro com um metro de altura e 1,81 centímetros de diâmetro (que corresponde ao diâmetro da menor árvore medida em campo). O limiar foi de aproximadamente 1000 pontos por tronco de árvore em nuvens de varredura múltipla e de 300 pontos por tronco de árvore em nuvens de varredura simples.

A aplicação do algoritmo segmentará a nuvem em subconjuntos de pontos quando houverem componentes conectadas, resultando no agrupamento de pontos dos objetos na cena de varredura, dentre eles: troncos, alvos e sub-bosque. A partir da segmentação foi possível obter o vetor de médias para cada subconjunto gerado conforme Equação 1,

$$
\bar{c}_{i}^{T}=\left[\bar{x}_{i} ; \bar{y}_{i} ; \bar{z}_{i}\right]
$$

em que ${ }^{c_{i}}$ é o vetor de médias das coordenadas dos pontos dos $n$ subconjuntos de dados em seus respectivos eixos $\left(\bar{x}_{i}, \bar{y}_{i}, \bar{z}_{i}\right)$.

3) Aplicação do algoritmo Tree Detecion in Plantation: O algoritmo Tree Detecion in Plantation demonstrado na Figura 3 e proposto neste trabalho, utiliza os vetores de médias ${ }^{\left(c_{i}\right.}, i$ $=1, \ldots, n$ ) obtidos na etapa de segmentação como referência para a análise do alinhamento de plantio para detecção de árvores em nuvem de pontos de TLS. 


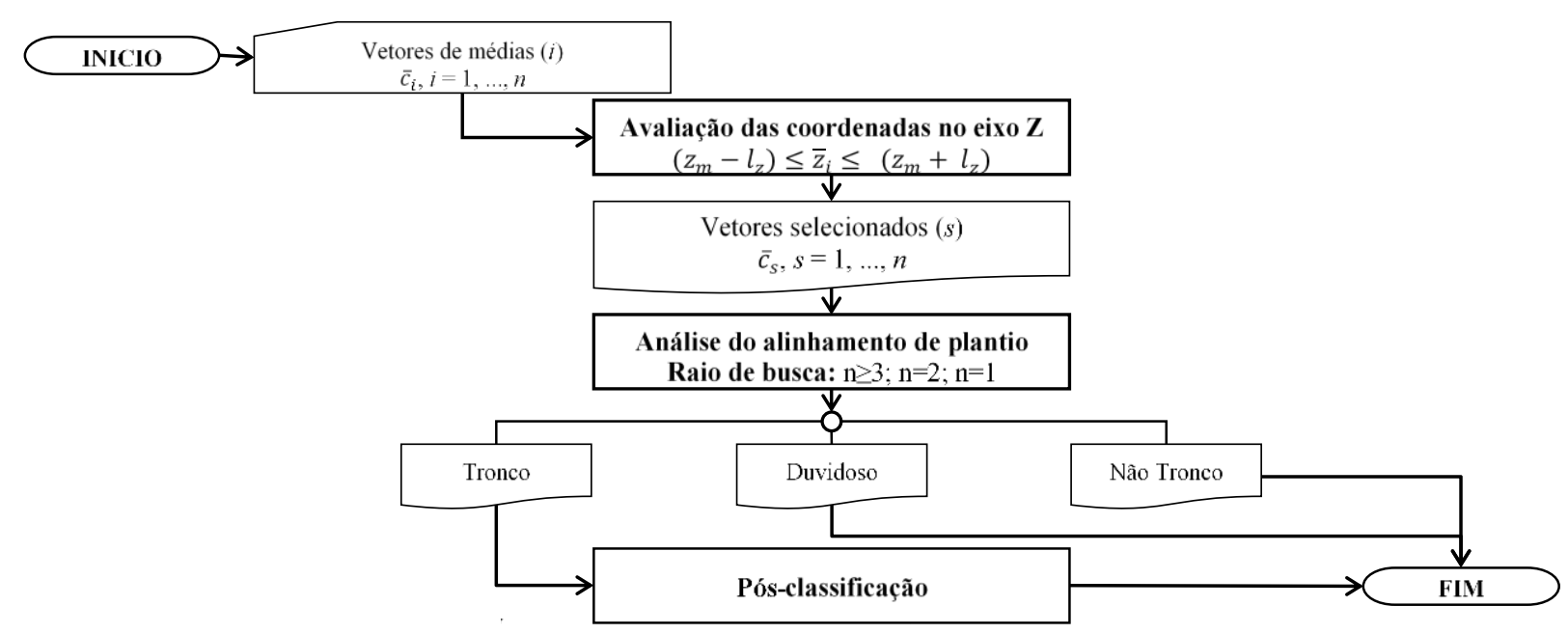

Figura 3: Algoritmo Tree Detection in Plantation (TDP)

As coordenadas dos vetores de médias são submetidas à aplicação de três procedimentos: a) Avaliação das coordenadas no eixo $\mathrm{Z}$; b) Análise do alinhamento de plantio; c) Pósclassificação. Como resultado os vetores são distinguidos em: Tronco; Duvidoso; Não tronco.

a) Avaliação das coordenadas no eixo $Z$ : A primeira análise a ser realizada avalia a coordenada vertical $\left({ }^{z}\right)$ dos vetores de médias $\left({ }^{c_{i}}\right)$ em relação ao centro do intervalo $\left(z_{m}\right)$ definido para o recorte da seção da nuvem de pontos pela Equação 2:

$$
z_{m}=\frac{1}{2}\left(z_{\max }-z_{\min }\right)
$$

em que $z_{m}$ é a média aritmética das cotas definidas para o recorte da seção da nuvem de pontos. Os vetores que apresentarem médias para coordenada vertical ( ${ }^{z}$ ) dentro de determinado intervalo definido pela Inequação 3 passam para próxima etapa de análise,

$$
\left(z_{m}-l_{z}\right) \leq \bar{z}_{\imath} \leq\left(z_{m}+l_{z}\right)
$$

em que $l_{z}$ é o limiar estabelecido para o desvio das médias em $\mathrm{Z}$ para os vetores analisados.

O limiar escolhido para o desvio da coordenada vertical do vetor de médias $\left(l_{z}\right)$ foi estabelecido em 0,20 metros. Espera-se que o valor definido para esse parâmetro seja suficiente para representar adequadamente o intervalo da média aritmética dos subconjuntos formado pelos troncos de árvores.

b) Análise do alinhamento de plantio: A análise do alinhamento é realizada a partir das coordenadas planas $(\mathrm{X} ; \mathrm{Y})$ dos vetores de médias selecionados ( ${ }_{s}{ }_{s}$ ) na etapa de avaliação das coordenadas do eixo Z. Cada vetor é analisado como um ponto de origem $\left(\mathrm{P}_{0}\right)$ em relação aos seus vizinhos dentro de um determinado raio de busca $R_{b}$ defino pela Equação 4: 


$$
R_{b}=E_{l}+l_{d}
$$

em que $R_{b}$ é o raio de busca a partir do ponto tomado como origem $\left(P_{0}\right), E_{l}$ é o espaçamento entre árvores na linha de plantio, e $l_{d}$ é o limite para desvios entre o espaçamento de árvores. Esse limite deve considerar o espaçamento na linha de plantio e uma distância máxima que uma árvore pode estar de sua vizinha. $\mathrm{O}$ limiar de 0,60 metros foi indicado para o espaçamento entre árvores $(2,20 \mathrm{~m})$ nas linhas dos plantios em questão.

A determinação dos azimutes e dos quadrantes que formam o alinhamento requer que no mínimo três vetores de médias $(n=3)$ sejam analisados em um sistema de coordenadas local, sendo um deles tomado como ponto de origem e dois como pontos vizinhos (Figura 4).

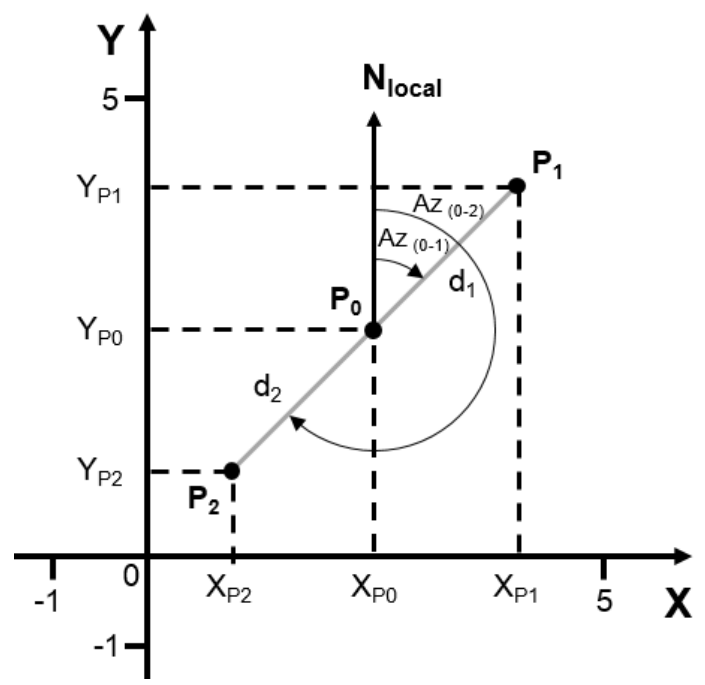

Figura 4: Projeção das distâncias e dos azimutes de um alinhamento entre três pontos.

* $\mathrm{P}_{0}$ - Ponto de origem; $\mathrm{P}_{1}$ e $\mathrm{P}_{2}-$ Pontos vizinhos; $\mathrm{d}_{1}$ e $\mathrm{d}_{2}$ - distâncias entre direções; $\mathrm{Az}(0-1)$ e $\mathrm{Az}_{(0-2)}-$ azimutes das direções.

A partir das coordenadas planimétricas dos três pontos é calculado o azimute das direções $\left(d_{l}\right.$ e $d_{2}$ ) tomando como origem o ponto central do alinhamento $\left(P_{0}\right)$. A diferença entre os azimutes calculados será o ângulo formado no alinhamento. Em povoamentos florestais é esperado que este se aproxime de $180^{\circ}$, conforme demonstrado pela Equação 5:

$$
A z_{p(0-2)}-A z_{p(0-1)} \cong 180^{\circ} \pm l_{a}
$$

em que $A z_{\mathrm{p}(0-1)}$ e $A z_{\mathrm{p}(0-2)}$ são os azimutes das direções formadas por um ponto de origem e seus vizinhos e $l_{a}$ é o limite do desvio angular para formação de um alinhamento no plantio. O limite para o desvio angular está relacionado com desvios na direção de alinhamento do plantio. Para o espaçamento de 2,20 metros foi estabelecido um limite de $10^{\circ}$, o que resulta em um desvio de aproximadamente $0,38 \mathrm{~m}$ da linha de plantio. Os pontos que se enquadram como alinhados são classificados de acordo com suas direções em seus respectivos quadrantes (Figura 5). 


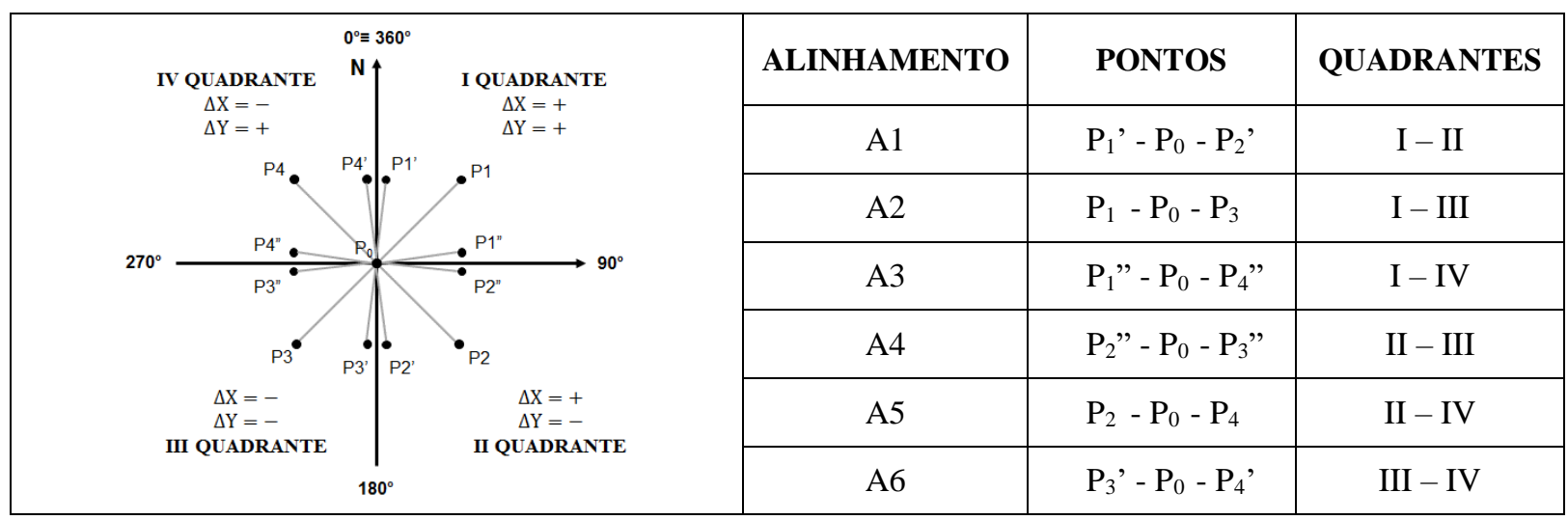

Figura 5: Classificação do alinhamento de acordo com as direções e quadrantes.

De acordo com a Figura 5, seis alinhamentos podem ser encontrados em povoamentos florestais. A definição do alinhamento se dá pela contagem dos alinhamentos que ocorreram um maior número de vezes no processamento. Nessa etapa também são obtidas as médias dos azimutes das duas direções que formam o alinhamento, conforme apresentado nas Equações 6 e 7:

$$
\begin{aligned}
& \overline{A z}_{d 1}=\frac{1}{n} \sum_{i=1}^{n} A z_{p(0-1)} \\
& \overline{A z}_{d 2}=\frac{1}{n} \sum_{i=1}^{n} A z_{P(0-2)}
\end{aligned}
$$

onde $A z_{d 1}$ e $A z_{d 2}$ correspondem à média dos azimutes das respectivas direções que formam o alinhamento considerando três pontos. Cabe ressaltar que em alinhamentos quadrados $(2 \times 2,3 \times 3)$ podem ocorrer alinhamentos nas linhas e entrelinhas de plantio. Nesse caso, o algoritmo deve assumir um dos alinhamentos como parâmetro para prosseguir a análise.

Após classificar o alinhamento e encontrar os azimutes das direções, os vetores de médias agora são analisados novamente quando três situações podem ocorrer dentro do raio de busca (Figura $6)$.

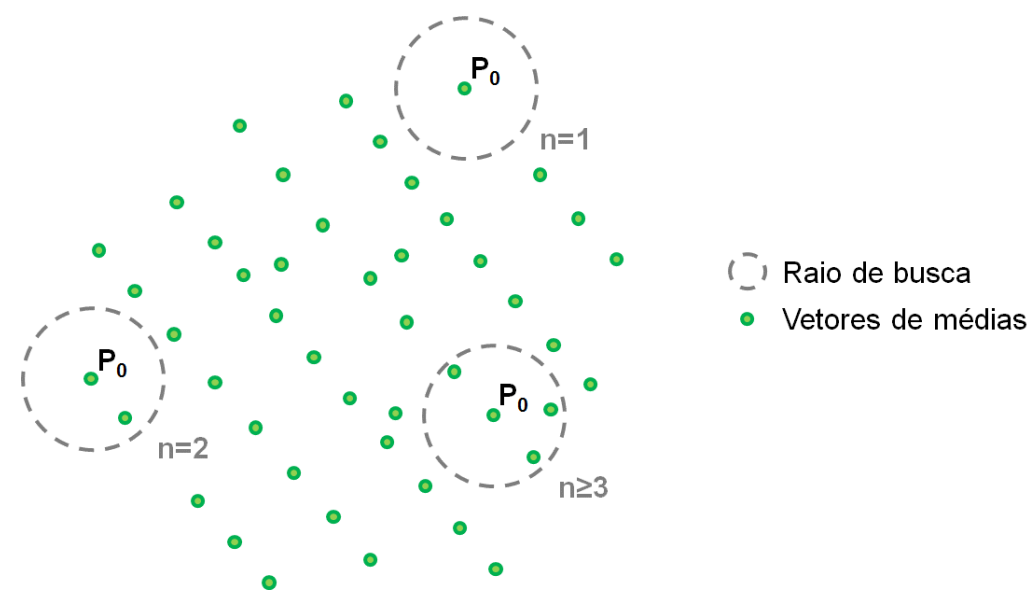

Figura 6: Raio de busca e vetores de médias 
* $n \geq 3$ : três ou mais vetores de médias; $n=2$ : dois vetores de médias; $n=1$ : um vetor de médias.

A partir das coordenadas planimétricas do vetor de médias definido como ponto de origem $\left(\mathrm{P}_{0}\right)$ são obtidos os azimutes e distâncias das direções formadas com seus vizinhos. Os vetores em análise são classificados como candidatos a tronco quando atendem as seguintes condições:

A primeira condição é que a distância da direção entre os pontos que estabelecem os azimutes esteja dentro de um intervalo definido pela Inequação 8 para distância entre árvores,

$$
\left(E_{l}-l_{d}\right) \leq d_{p(0-i)} \leq\left(E_{l}+l_{d}\right)
$$

em que $d_{p(0-\mathrm{i})}$ é a distância da direção entre o ponto de origem e seu vizinho, $E_{l}$ é o espaçamento entre árvores na linha de plantio, e $l_{d}$ é o limite para desvios entre o espaçamento de árvores no plantio.

A segunda condição é que o azimute desta direção corresponda a aproximadamente o azimute médio para o quadrante do alinhamento em análise, como demonstrado na Inequação 9:

$$
\left(\overline{A z}_{d i}-l_{a}\right) \leq A z_{p(0-i)} \leq\left(\overline{A z}_{d i}+l_{a}\right)
$$

em que $A z_{\mathrm{p}(0-\mathrm{i})}$ é o azimute da direção $i,{ }^{A z_{d i}}$ corresponde à média dos azimutes para o respectivo quadrante definido no alinhamento, e $l_{a}$ é o limite do desvio angular para formação de um alinhamento.

Inicialmente esse procedimento é aplicado para três ou mais vetores de médias $(\mathrm{n} \geq 3)$ e em seguida para dois vetores de médias $(\mathrm{n}=2)$. A análise de dois vetores é realizada quando o ponto de origem $\left(\mathrm{P}_{0}\right)$ ainda não foi classificado como candidato a tronco e apresenta apenas um vizinho dentro do raio de busca pré-estabelecido. Nessa etapa, o ponto que apresentar apenas um vizinho e não satisfizer as condições será classificado como "duvidoso". No entanto, se o seu vizinho foi previamente classificado como candidato a tronco, ele será classificado como "Não tronco".

A análise de apenas um vetor de médias $(\mathrm{n}=1)$ é realizada quando um ponto de origem $\left(\mathrm{P}_{0}\right)$ não possui vizinhos dentro do raio de busca pré-estabelecido. A ocorrência de pontos isolados pode indicar falhas no plantio e árvores limítrofes na parcela. Dessa forma, os pontos isolados são classificados como duvidosos, e devem ser submetidos a análise visual para que possam ser aceitos como candidatos a tronco ou eliminados como não troncos.

c) Pós classificação: A pós classificação é realizada para verificar a proximidade entre os vetores de médias selecionados como troncos. Se a distância entre dois vetores de médias for menor ou igual ao limiar pré-estabelecido, os vetores são classificados como duvidosos, como definido na Inequação 10:

$$
d_{p(0-1)} \leq l_{d}
$$


em que $d_{p(0-1)}$ é a distância do ponto de origem e seu vizinho, e $l_{d}$ é o limiar aceitável para o espaçamento entre árvores no plantio.

\subsection{Avaliação dos resultados}

Os resultados para detecção de árvores em nuvens de pontos de parcelas circulares em povoamentos de Eucalyptus spp. foram avaliados de acordo com os seguintes critérios: número de árvores contadas em campo; interpretação visual nas varreduras simples e múltiplas para contagem do número de árvores; detecções automáticas; detecções duvidosas; Erro Tipo I e Erro tipo II. O Erro tipo I corresponde as árvores que estão presentes na nuvem de pontos, mas não foram detectadas. O Erro tipo II corresponde a objetos detectados erroneamente como árvores.

\section{RESULTADOS E DISCUSSÃO}

\subsection{Validação do número de árvores interpretadas visualmente em varreduras simples e múltiplas}

Os resultados indicaram subestimativas quanto ao número de árvores interpretadas visualmente em nuvens de pontos de parcelas circulares com área de $400 \mathrm{~m}^{2}$ levantadas por varredura simples, em que foram identificadas em média $95 \%$ das árvores para idade de 5 anos e $96 \%$ para as idades de 4 e 2 anos. Em contrapartida, 100\% das árvores demarcadas em campo foram identificadas nas nuvens de pontos geradas a partir de varredura múltipla (Tabela 1).

Tabela 1: Número de árvores em campo e em nuvem de pontos de TLS

\begin{tabular}{|c|c|c|c|c|c|c|c|c|c|c|c|c|}
\hline \multirow{2}{*}{ PARCELAS } & \multicolumn{4}{|c|}{5 anos } & \multicolumn{4}{|c|}{4 anos } & \multicolumn{4}{|c|}{2 anos } \\
\hline & $\mathbf{1}$ & 2 & 3 & 4 & 5 & 6 & 7 & 8 & 9 & 10 & 11 & 12 \\
\hline ÁRVORES EM CAMPO & 33 & 38 & 41 & 40 & 49 & 51 & 48 & 47 & 41 & 46 & 41 & 41 \\
\hline INTERPRETAÇÃO VISUAL (VS) & 31 & 37 & 38 & 38 & 49 & 49 & 47 & 42 & 40 & 44 & 40 & 39 \\
\hline INTERPRETAÇÃO VISUAL (VM) & 33 & 38 & 41 & 40 & 49 & 51 & 48 & 47 & 41 & 46 & 41 & 41 \\
\hline
\end{tabular}

*VS = varredura simples; $\mathrm{VM}=$ varredura múltipla.

A distribuição espacial das árvores em nuvens de pontos de varredura múltipla foi demonstrada graficamente para que fosse possível determinar a localização das árvores sombreadas em levantamentos por varredura simples (Figura 7). 

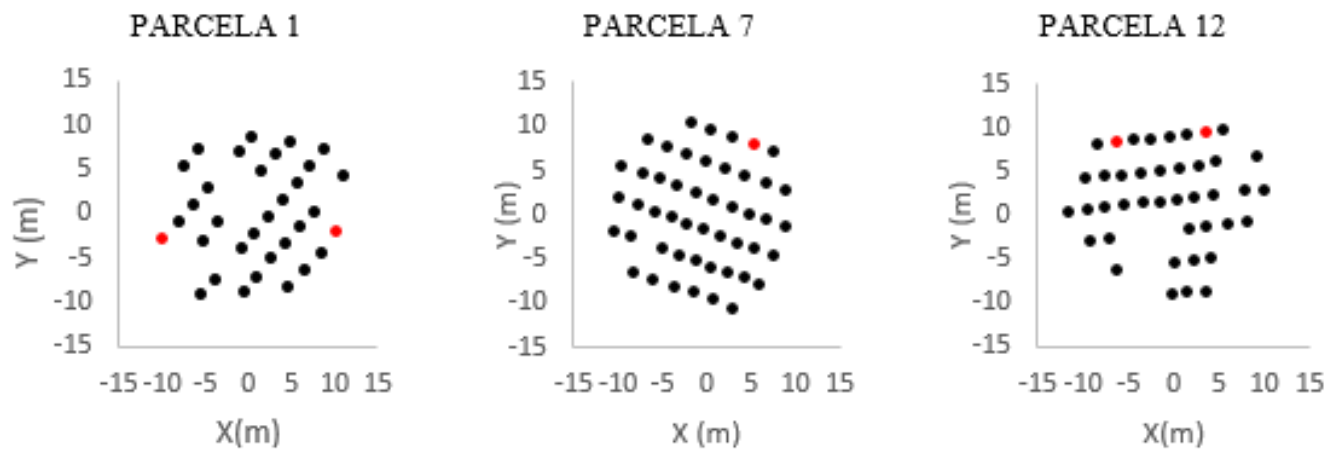

- Projeção das árvores em nuvem de pontos - Árvores sombreadas na varredura simples

Figura 7: Distribuição espacial das árvores em nuvens de pontos de parcelas circulares $\left(400 \mathrm{~m}^{2}\right)$

De acordo com representação da distribuição espacial é possível inferir que árvores localizadas nos limites da parcela de $400 \mathrm{~m}^{2}$ representam a maior incidência de árvores sombreadas. Considerando a distribuição espacial das árvores nos povoamentos estudados foi constatado que a partir de aproximadamente 5 metros do equipamento laser terrestre podem ocorrer sombreamento de árvores na varredura simples. Apesar do sombreamento ter sido constatado em todas as idades dos povoamentos estudados, sua ocorrência foi maior nas parcelas dos povoamentos de 4 e 5 anos, fato que pode ser atribuído ao maior diâmetro das árvores nessas parcelas (Figura 8).

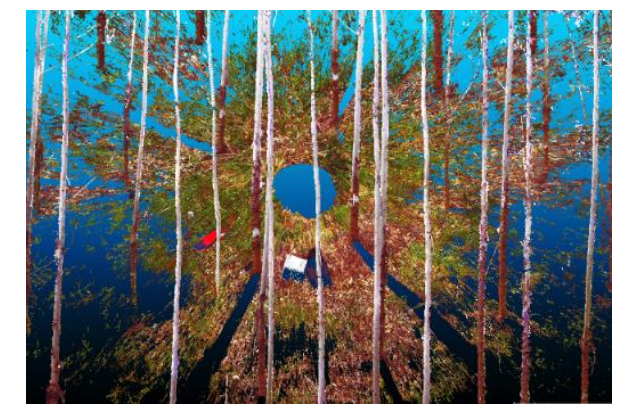

(A)

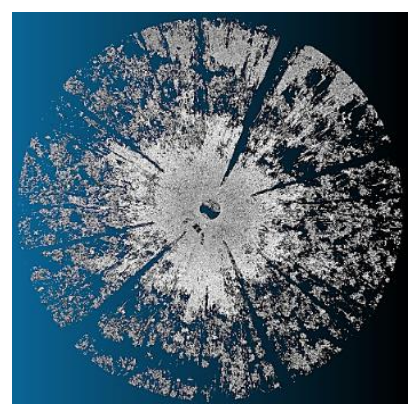

(B)

Figura 8: Efeito do sombreamento na varredura simples em parcela circular de $400 \mathrm{~m}^{2}$ no povoamento de 5 anos de idade: (A) vista frontal; (B) vista de topo.

O efeito do sombreamento é um fator que afeta a acurácia das estimativas das variáveis dendrométricas em parcelas, aumentando o risco de erros sistemáticos associados à subestimação do número de árvores existentes na parcela (Zasada et al., 2013). As taxas de detecção são influenciadas pelo efeito de sombreamento em varreduras simples. Uma vez que a magnitude desse efeito depende da estrutura dos povoamentos e características do local, é importante relatar taxas de detecção com base em árvores potencialmente detectáveis na varredura, a fim de proporcionar resultados para comparação objetiva com outros métodos baseados em varredura simples (Pueschel et al., 2013). 


\subsection{Segmentação da nuvem de pontos}

A partir da segmentação por componentes conectadas as coordenadas dos vetores de médias $\left(\bar{c}_{i}\right)$ foram utilizadas para reconstruir a distribuição espacial das árvores em cada parcela com objetivo de projetar o padrão do alinhamento (Figura 9 e 10).
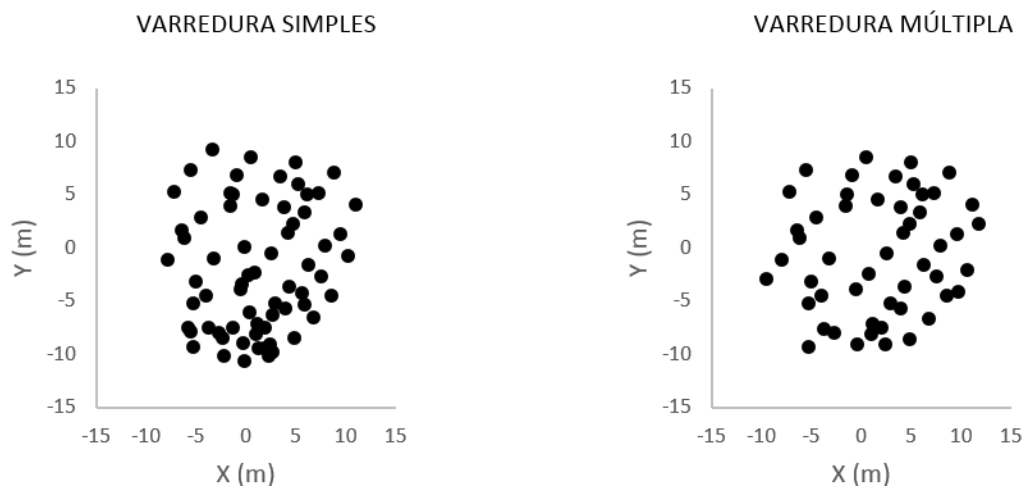

Figura 9: Projeção das coordenadas XY dos vetores de média: Parcela 1
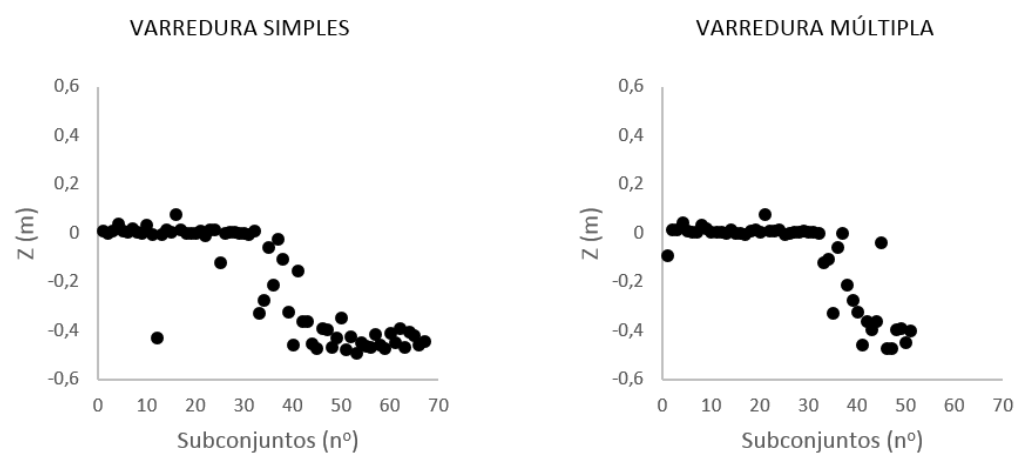

Figura 10: Projeção da coordenada $Z$ dos vetores de médias: Parcela 1

No entanto, apenas o processo de segmentação não garante que todos os subconjuntos gerados representam troncos de árvores, outros objetos em campo podem estar presentes, como por exemplo, os tripés e alvos localizados entre linhas de plantio, arbustos e plantas do sub-bosque.

\subsection{Detecção de árvores}

$\mathrm{A}$ avaliação da coordenada no eixo $\mathrm{Z}$ resultou na seleção dos vetores de médias $\left(\bar{c}_{S}\right)$ que se encontravam dentro do intervalo definido $\left(-0,20 m \leq z_{l} \leq+0,20 m\right)$ e assim esses foram considerados nas próximas etapas de análise. Os vetores de médias que estavam fora desse intervalo foram eliminados (Figura 11). 

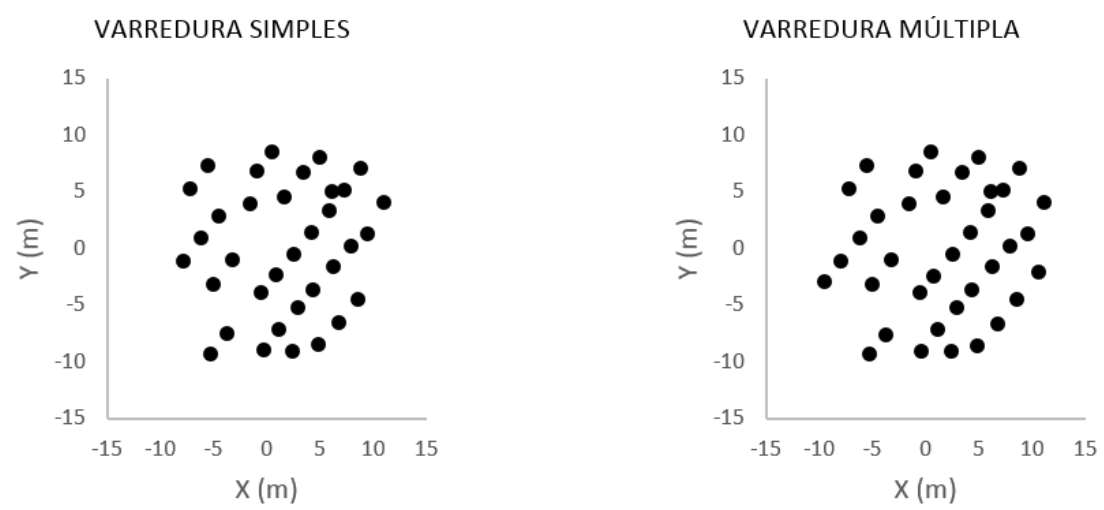

Figura 11: Representação das coordenadas XY dos vetores de médias selecionados a partir do limiar estabelecido para coordenada Z: Parcela 1

A eliminação de vetores de médias que não representam árvores na cena de varredura foi fundamental para que a distribuição espacial caracterizasse o padrão de alinhamento dos plantios.

A detecção automática de árvores e detecções duvidosas indicadas pelo algoritmo TDP foram avaliadas quanto ao número de árvores que visualmente estavam presentes nas nuvens de pontos de varreduras simples e múltiplas (Tabela 2 e 3).

Tabela 2: Resultados da detecção de árvores na varredura simples

\begin{tabular}{|c|c|c|c|c|c|c|c|c|c|c|c|c|}
\hline \multirow{2}{*}{ PARCELA } & \multicolumn{4}{|c|}{5 anos } & \multicolumn{4}{|c|}{4 anos } & \multicolumn{4}{|c|}{2 anos } \\
\hline & 1 & 2 & 3 & 4 & 5 & 6 & 7 & 8 & 9 & 10 & 11 & 12 \\
\hline INTERPRETAÇÃO VISUAL & 31 & 37 & 38 & 38 & 49 & 48 & 47 & 42 & 40 & 44 & 40 & 39 \\
\hline DETECÇÃO AUTOMÁTICA & 30 & 31 & 31 & 36 & 48 & 48 & 46 & 37 & 39 & 42 & 38 & 35 \\
\hline DETECÇÕES DUVIDOSAS & 3 & 7 & 10 & 2 & 1 & 1 & 2 & 7 & 1 & 2 & 2 & 6 \\
\hline Troncos & 1 & 6 & 8 & 2 & 1 & 0 & 1 & 5 & 1 & 2 & 2 & 5 \\
\hline Objetos & 2 & 1 & 2 & 0 & 0 & 1 & 1 & 2 & 0 & 0 & 0 & 1 \\
\hline ERRO TIPO I & - & - & - & - & - & - & - & - & - & - & - & - \\
\hline ERRO TIPO II & - & - & 1 & - & - & - & - & - & - & - & - & 1 \\
\hline
\end{tabular}

Tabela 3: Resultados da detecção de árvores na varredura múltipla

\begin{tabular}{lccccc|cccc|ccc}
\hline \multirow{2}{*}{ PARCELA } & \multicolumn{3}{c}{$\mathbf{5}$ anos } & \multicolumn{4}{c|}{$\mathbf{4}$ anos } & \multicolumn{3}{c}{$\mathbf{2}$ anos } \\
\cline { 2 - 14 } & 1 & 2 & 3 & 4 & 5 & 6 & 7 & 8 & 9 & 10 & 11 & 12 \\
\hline INTERPRETAÇÃO VISUAL & 33 & 38 & 41 & 40 & 49 & 51 & 48 & 47 & 41 & 46 & 41 & 41 \\
\hline DETECÇÃO AUTOMÁTICA & 31 & 33 & 36 & 38 & 48 & 51 & 48 & 46 & 40 & 45 & 38 & 38 \\
\hline DETECÇÕES DUVIDOSAS & 4 & 6 & 7 & 2 & 1 & 1 & 0 & 1 & 1 & 1 & 4 & 5 \\
\hline \multicolumn{1}{c}{ Troncos } & 2 & 5 & 6 & 2 & 1 & 0 & 0 & 1 & 1 & 1 & 3 & 3 \\
\hline \multicolumn{1}{c}{ Objetos } & 2 & 1 & 1 & 0 & 0 & 1 & 0 & 0 & 0 & 0 & 1 & 2 \\
\hline ERRO TIPO I & - & - & - & - & - & - & - & - & - & - & - & - \\
\hline ERRO TIPO II & - & - & 1 & - & - & - & - & - & - & - & - & - \\
\hline
\end{tabular}

De acordo com os resultados não foram encontrados Erros tipo I no processamento dos dados de nuvens de pontos de varreduras simples e múltiplas, e isso pode ser explicado pela possibilidade de os pontos serem classificados como duvidosos. Os Erros tipo II foram encontrados nas nuvens de pontos de varreduras simples e múltiplas, como por exemplo, na Parcela 03 (Figura 12). 

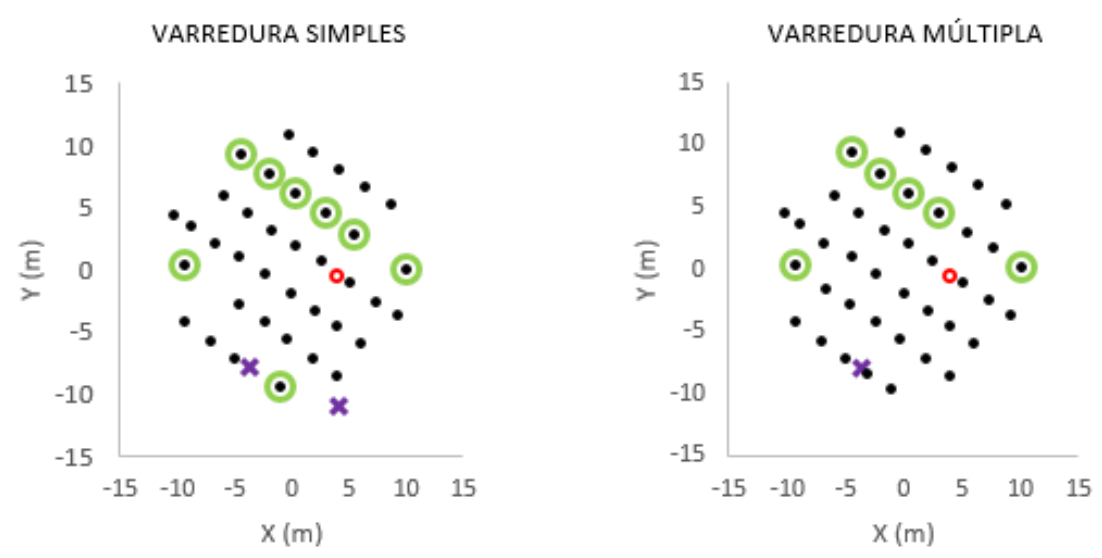

- Árvores da parcela $\bigcirc$ Duvidosa: Tronco

X Duvidosa: Objeto O Erro tipo II

Figura 12: Coordenadas XY dos vetores de média classificados pelo algoritmo TDP: Parcela 03

localização do objeto classificado como Erro tipo II está alinhada entre dois vetores de médias que representam árvores e dentro do limiar estabelecido para distância entre os pontos, ensejando que o algoritmo incida no erro. O objeto em questão trata-se do tripé onde foi instalado um alvo em campo para o registro das varreduras. Esse tipo de erro pode ser suprimido se na etapa de registro os alvos e acessórios utilizados para implantação dos pontos de controle forem eliminados após sua utilização. Para avaliar o desempenho do algoritmo optou-se por mantê-los.

A quantidade de árvores classificadas como duvidosas na parcela 3 foi investigada. Procedeu-se o cálculo da distância entre os pontos e foi verificado que para a parcela em questão, as árvores alinhadas foram consideradas como isoladas porque estavam fora do intervalo estabelecido para distância entre árvores no plantio.

Os objetos classificados como duvidosos que não são árvores também podem ser observados na parcela 3 (Figura 12). A sua ocorrência está ligada à presença de pontos isolados e também pontos alinhados com árvores no plantio, tais características também foram observadas nas demais parcelas estudas.

A ocorrência de Erros tipo II e de árvores duvidosas que são troncos ocorreram também na parcela 12 em nuvem de pontos de varredura simples (Figura 13).
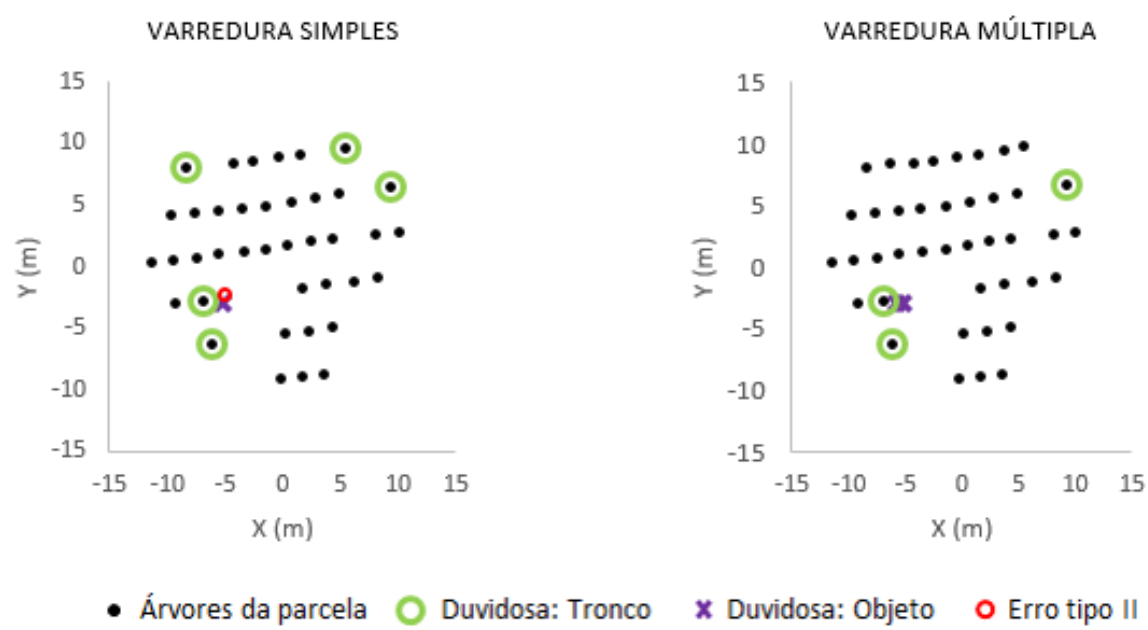

Figura 13: Coordenadas XY dos vetores de média classificados pelo algoritmo TDP: Parcela 12 
O objeto segmentado encontrava-se dentro das condições impostas para a ocorrência de uma árvore, dessa forma, foi classificado erroneamente ao se utilizar a varredura simples. Já na varredura múltipla esse erro não aconteceu porque os pontos foram classificados como duvidosos. A ausência de árvores observadas na parcela 12 (Figura 13) são falhas no plantio.

Os melhores resultados foram observados na parcela 7. Apenas uma árvore e um objeto foram classificados como duvidosos na varredura simples. O objeto duvidoso trata-se de um galho espesso que foi segmentado e classificado como duvidoso na etapa de detecção, essa situação poderá ser comum quando da ocorrência de árvores bifurcadas. Já na varredura múltipla, todas as árvores foram detectadas automaticamente.

Os resultados da classificação automática em conjunto com a verificação visual corresponderam à identificação de $100 \%$ das árvores nas parcelas, eliminando Erros tipo I. Simonse et al. (2003) encontraram correspondência de cerca de $90 \%$ dos indivíduos identificados e Bienert et al. (2007) identificaram cerca de $97 \%$ de árvores automaticamente no processamento da nuvem de pontos. Os autores relataram que árvores não identificadas estavam encobertas por pequenos galhos ou sombreadas por outras árvores.

Sabe-se que a alocação do equipamento em campo afeta diretamente a detecção das árvores. Liang et al. (2011) concluíram que, em distância de até 15 metros do laser terrestre, todas as árvores em uma área dominada por Pinus sylvestris L. foram identificadas corretamente pelo método automatizado. A partir de 15 metros e até 60 metros, dividindo os resultados em classes de 5 e $10 \mathrm{~m}$ de amplitude, cerca de $70 \%$ das árvores foram encontradas automaticamente, sendo a detecção manual considerada como parâmetro de comparação. O sombreamento de árvores na varredura simples é apontado como o principal problema na detecção dos indivíduos.

\section{Conclusões}

O presente estudo demonstrou que ao se trabalhar com nuvens de pontos geradas a partir da varredura simples em parcelas circulares, convencionalmente adotada pelas empresas florestais, podem ocorrer subestimativas quanto ao número de árvores por unidade de área. A identificação de árvores em nuvens de pontos de varreduras simples foi de $95 \%$ para idade de 5 anos e $96 \%$ para as idades de 4 e 5 anos. O efeito de sombreamento é apontado como a razão desse problema, sendo possível sua eliminação empregando múltiplas varreduras, $100 \%$ das árvores são identificadas.

Ao se trabalhar com o número real de árvores identificadas em nuvens de pontos TLS foi possível constatar que a abordagem semiautomática proposta neste estudo para a detecção de árvores resultou em valores acurados tanto para as parcelas levantadas por varredura simples quanto para as de varreduras múltiplas. A possibilidade de classificar os vetores de médias como duvidosos tornou o algoritmo robusto o suficiente para que não ocorressem erros de eliminação de árvores.

Os erros de inclusão, representados por objetos classificados como árvores, podem ser atribuídos aos acessórios utilizados no levantamento, galhos espessos e ao sub-bosque contemplado na seção da nuvem de pontos da parcela. Cabe ressaltar que os acessórios podem ser excluídos da nuvem de pontos na etapa de registro, contribuindo para uma melhoria dos resultados. 
A partir da detecção de troncos em nuvem de pontos é possível isolar as árvores do plantio e aplicar técnicas de modelagem tridimensional. A utilização da varredura laser terrestre representa uma quebra de paradigma na coleta de dados para fins de inventário florestal em plantios com espaçamentos regulares. O presente estudo relatou sua aplicação em parcelas circulares, no entanto é possível vislumbrar novos estudos a partir de dados laser que não estejam condicionados a essa forma de unidade amostral, na qual o algoritmo proposto poderá ser aplicado.

Estudos sobre o melhor posicionamento do equipamento em campo, a resolução adequada para obtenção de variáveis dendrométricas e o desenvolvimento de métodos de amostragem, ainda se fazem necessários. O aprimoramento de técnicas de levantamento em um ambiente florestal bem como o desenvolvimento de algoritmos robustos que possam superar as limitações encontradas no levantamento e processamento dos dados tridimensionais representam os desafios a serem superados para utilização da varredura laser terrestre para mensuração em plantios florestais comerciais.

\section{AGRADECIMENTOS}

Agradecemos a Eldorado do Brasil pelo apoio ao projeto de pesquisa e a Santiago \& Cintra pela disponibilização do equipamento Trimble TX5 e suporte técnico à coleta de dados.

\section{REFERÊNCIAS BIBLIOGRÁFICAS}

Aschoff, T., Spiecker, H. "Algorithms for the automatic detection of trees in laser scanner data." International Archives of Photogrammetry, Remote Sensing and Spatial Information Sciences 36.Part 8 (2004): W2.

Aschoff, T., Thies, M., Spiecker, H. "Describing forest stands using terrestrial laserscanning." International Archives of Photogrammetry, Remote Sensing and Spatial Information Sciences 35.5 (2004): 237-241.

Bienert, A., Scheller, S., Keane, E., Mullooly, G., and Mohan, F. "Application of terrestrial laser scanners for the determination of forest inventory parameters." International Archives of Photogrammetry, Remote Sensing and Spatial Information Sciences 36.Part 5 (2006).

Bienert, A., Scheller, S., Keane, E., Mohan, F., and Nugent, C. "Tree detection and diameter estimations by analysis of forest terrestrial laserscanner point clouds." ISPRS workshop on laser scanning. Vol. 2007. 2007.

Bienert, A., Queck, R., Schmidt, A., Bernhofer, C., and Maas, H. G. "Voxel space analysis of terrestrial laser scans in forests for wind field modelling." International Archives of Photogrammetry, Remote Sensing and Spatial Information Sciences 38.Part 5 (2010): 92-97.

Brolly, G., Kiraly, G. "Algorithms for stem mapping by means of terrestrial laser scanning." Acta Silvatica et Lignaria Hungarica 5 (2009): 119-130.

Bucksch, A., Appel van Wageningen, H. "Skeletonization and segmentation of point clouds using octrees and graph theory." Proceedings of Commission V Symposium, Image Engineering and Vision Metrology. 2006. 
Bucksch, A., Lindenbergh, R., Menenti, M. "SkelTre."The Visual Computer 26.10 (2010): 12831300 .

Girardeau-Montaut, Daniel. Détection de changement sur des données géométriques tridimensionnelles. Diss. Télécom ParisTech, 2006.

Gorte, B., Pfeifer, N. "Structuring laser-scanned trees using 3D mathematical morphology." International Archives of Photogrammetry and Remote Sensing 35.B5 (2004): 929-933.

Gorte, B., Winterhalder, D. "Reconstruction of laser-scanned trees using filter operations in the 3D raster domain." International Archives of Photogrammetry, Remote Sensing and Spatial Information Sciences 36.Part 8 (2004): W2.

Liang, X., Hyyppä, J. "Automatic stem mapping by merging several terrestrial laser scans at the feature and decision levels." Sensors13.2 (2013): 1614-1634.

Liang, X., Litkey, P., Hyyppä,J., Kaartinen, H., Vastaranta, M., Holopainen, M. "Automatic stem mapping using single-scan terrestrial laser scanning." Geoscience and Remote Sensing, IEEE Transactions on50.2 (2012): 661-670.

Liang, X., Litkey, P., Hyyppä, J., Kaartinen, H., Kukko, A., Holopainen, M. "Automatic plotwise tree location mapping using single-scan terrestrial laser scanning." Photogramm. J. Finland 22.2 (2011): 37-48.

Meagher, D. "Geometric modeling using octree encoding." Computer graphics and image processing 19.2 (1982): 129-147.

Pueschel, P., Newnham, G., Rock, G., Udelhoven, T., Werner, W., Hill, J. "The influence of scan mode and circle fitting on tree stem detection, stem diameter and volume extraction from terrestrial laser scans." ISPRS Journal of Photogrammetry and Remote Sensing 77 (2013): 44-56.

Simonse, M., Aschoff, T., Spiecker, H., Thies,M. "Automatic determination of forest inventory parameters using terrestrial laserscanning." Proceedings of the ScandLaser Scientific Workshop on Airborne Laser Scanning of Forests. Vol. 2003. 2003.

Schilling, A., Schmidt, A., Maas, H. "Automatic Tree Detection and Diameter Estimation in Terrestrial Laser Scanner Point Clouds." Proceedings of the 16th Computer Vision Winter Workshop. 2011.

Zasada, M., Steren'czak, K., Dudek, W.M., Rybski, A. "Horizon visibility and accuracy of stocking determination on circular sample plots using automated remote measurement techniques." Forest ecology and management 302 (2013): 171-177.

Recebido em 05 de setembro de 2015.

Aceito em 10 de agosto de 2016. 